\title{
Universally finitary symbolic extensions
}

by

\author{
Jacek Serafin (Wrocław)
}

\begin{abstract}
We prove that by considering a finitary (almost continuous) symbolic extension of a topological dynamical system instead of a continuous extension, one cannot achieve any drop of the entropy of the extension.
\end{abstract}

1. Introduction. Symbolic extension entropy has been an object of intensive studies for two decades now. Roughly speaking, the symbolic extension entropy of a given topological dynamical system is the infimum of the topological entropies over the set of all symbolic dynamical systems for which the original system is a factor; here the map bonding the systems is continuous. Recently Downarowicz asked if it was possible to obtain a symbolic system which would be an almost-continuous (finitary) extension of a given topological dynamical system and have entropy strictly smaller than the entropy of any true (continuous) symbolic extension. In this short note we answer Downarowicz's question in the negative.

Our proof substantially uses the concept of asymptotic h-expansiveness, introduced by Misiurewicz [5] in his study of conditions for the existence of measures of maximal entropy. The notion of asymptotic h-expansiveness has also been fundamental for most constructions of symbolic extensions of topological dynamical systems. The interested reader is referred to [1] for an extended discussion of entropy of symbolic extensions.

2. Preliminaries and notation. Most of our notation coincides with that of [1]. A subshift (or symbolic system) is the action of the left shift transformation $S$ on a closed and shift-invariant subset $Y$ of $\Lambda^{\mathbb{Z}}$, the collection of doubly-infinite sequences over a finite alphabet $\Lambda$.

Let $X$ be a compact, metrizable topological space and $T$ be a homeomorphism of $X$. The pair $(X, T)$ is called a topological dynamical system. We

2010 Mathematics Subject Classification: 37A35, 37B10, 37B40.

Key words and phrases: symbolic extension, entropy, finitary mapping. 
shall assume that the topological entropy $h_{\text {top }}(X)$ of $(X, T)$ is finite. It is standard to denote by $\mathcal{M}(X, T)$ the set of Borel probability measures on $X$, invariant with respect to $T$. It is well-known that this set is compact in the weak ${ }^{\star}$ topology.

If a topological system $(X, T)$ is a factor of a symbolic system $(Y, S)$ via a continuous mapping $\phi: Y \rightarrow X$, intertwining the actions $T$ and $S$, i.e. $\phi \circ S=T \circ \phi$, then we say that $(Y, S)$ is a symbolic extension of $(X, T)$, and we call the bonding map $\phi$ equivariant.

The extension entropy function of a continuous extension $\phi: Y \rightarrow X$ is a function on $\mathcal{M}(X, T)$ defined as follows:

$$
h_{\mathrm{ext}}^{\phi}(\mu)=\sup \{h(S, \nu): \nu \in \mathcal{M}(Y, S) \text { and } \phi \nu=\mu\},
$$

where $\phi: \mathcal{M}(Y, S) \rightarrow \mathcal{M}(X, T)$ is the surjection defined naturally by $\phi \nu(A)$ $=\nu\left(\phi^{-1} A\right)(A \subset X)$. One then defines the symbolic extension entropy function of $T$ as

$$
h_{\text {sex }}(T, \mu)=\inf \left\{h_{\text {ext }}^{\phi}(\mu): \phi \text { is a symbolic extension of } T\right\} .
$$

Recall here that an extension $\phi$ is called principal if $h_{\text {ext }}^{\phi}(\mu)=h(\mu)$ for every $\mu \in \mathcal{M}(X, T)$.

The above definitions make sense also if $\phi$ is not necessarily continuous; it is sufficient that $\phi$ is measurable and equivariant. In this spirit, we want to know if weakening the continuity assumption has any consequences on the entropy of symbolic extension, and so we proceed with the following definition.

Definition 1. If $(X, T)$ and $(Y, S)$ are two topological systems and $\phi$ is a mapping from $Y$ to $X$ then we call $\phi$ universally finitary if the following conditions hold:

- $\phi$ intertwines the actions $T$ and $S$,

- there exist subsets $Y_{C} \subset Y$ and $X_{C} \subset X$ such that: $Y_{C}$ is dense in $Y$ and $X_{C}$ is dense in $X ; Y_{C}$ and $X_{C}$ are full sets (of measure 1) with respect to all invariant measures on $Y$ and $X$, respectively; and $\phi: Y_{C} \rightarrow X_{C}$ is a continuous surjection.

Let us note that our definition of universal finitariness differs slightly from a classical definition of finitariness (which is often referred to as almost continuity). In the classical approach one fixes an invariant measure $\mu$ on $X$ and $\nu$ on $Y$ and the sets $X_{C}$ and $Y_{C}$ are required to be of measure 1 with respect to $\mu$ and $\nu$ respectively. We require the same for all invariant measures on respective spaces, which justifies the adverb "universally". We skip the standard assumption that the sets $X_{C}$ and $Y_{C}$ are residual. It is not hard to see that if $X_{C}$ and $Y_{C}$ are dense then by modifying the map outside $Y_{C}$ one can obtain a continuous surjection between residual sets $X_{C}^{\prime}$ and 
$Y_{C}^{\prime}$ containing $X_{C}$ and $Y_{C}$, respectively. This, however, plays no role in our argument. For a detailed account of almost continuity we refer the reader to [3].

We shall prove that the quantity $h_{\mathrm{sex}}(T, \mu)$ remains unchanged if one considers universally finitary symbolic extensions instead of symbolic extensions.

3. Improving finitary extensions. We now prove that a universally finitary extension can be modified to a continuous extension without any increase of entropy.

Theorem 1. Let $(X, T)$ be an arbitrary, finite entropy topological system. Suppose $(Y, S)$ is a universally finitary symbolic extension of $(X, T)$ via a mapping $\phi$. Then there exists a symbolic dynamical system $\left(Y^{\prime}, S^{\prime}\right)$ such that

- $\left(Y^{\prime}, S^{\prime}\right)$ is a continuous extension of $(X, T)$ via a mapping $\psi$,

- $\left(Y^{\prime}, S^{\prime}\right)$ is a principal extension of $(Y, S)$ via a mapping $\pi$,

- $\psi=\phi \circ \pi$ on $\mathcal{M}\left(Y^{\prime}, S^{\prime}\right)$.

In particular, this implies that $h_{\mathrm{ext}}^{\phi} \equiv h_{\mathrm{ext}}^{\psi}$ on $\mathcal{M}(X, T)$.

Proof. Suppose that $(Y, S)$ is a universally finitary symbolic extension of $(X, T)$ via the mapping $\phi$. Set

$$
\Phi=\left\{(x, y) \in X \times Y: x=\phi(y), y \in Y_{C}\right\},
$$

the graph of the mapping $\left.\phi\right|_{Y_{C}}$. Then define

$$
Z=\bar{\Phi}
$$

the closure of $\Phi$ in the product topology. The action on $Z$ is $\left.(T \times S)\right|_{Z}$. Clearly, $Z$, being the closure of an invariant set, is invariant. Let $\pi_{X}, \pi_{Y}$ denote the (continuous) projections of $Z$ into $X$ and $Y$. The sets $\pi_{X}(Z)$ and $\pi_{Y}(Z)$ are both dense (they contain $X_{C}$ and $Y_{C}$, respectively) and closed (as continuous images of the compact set $Z$ ), hence $\pi_{X}$ and $\pi_{Y}$ are surjections onto $X$ and $Y$, respectively. Consider the following, non-commuting, diagram:

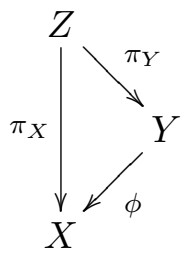

Define $D_{Z}=\{(x, y) \in Z: x \neq \phi(y)\}$ and observe that $D_{Z}$ is a universally null set, i.e. $\xi\left(D_{Z}\right)=0$ for every Borel probability measure $\xi$, invariant with respect to $T \times S$. In order to see that, consider a multifunction

$$
y \mapsto\{x \in X:(x, y) \in Z\}
$$


which assigns to each $y \in Y$ the $y$-section of $Z$. Note that if $y \in Y_{C}$ then by continuity of $\phi$, the section over $y$ consists of a single point $x=\phi(y)$, hence $(x, y) \in Z \backslash D_{Z}$. Consequently, we have

$$
D_{Z} \subset \pi_{Y}^{-1}\left(Y \backslash Y_{C}\right)
$$

recall here that $Y \backslash Y_{C}$ is, by assumption, a zero-measure set for all invariant measures on $Y$. As the projection of an invariant measure is again an invariant measure, it follows that $D_{Z}$ is a universally null set and we have

$$
\xi\{(x, y) \in Z: x=\phi(y)\}=1
$$

for every $\xi \in \mathcal{M}(Z, T \times S)$. The above condition means that the projection $\pi_{Y}$ is an invertible map on the universally full set $Z \backslash D_{Z}$, and that clearly implies that

$$
h(T \times S, \xi)=h(S, \eta),
$$

where $\nu$ is the projection of $\xi$ onto $Y$. We have shown that $Z$ is a principal (but not necessarily symbolic) extension of $Y$.

Further, as $Z$ is a principal extension of a symbolic system $(Y, S)$, it is asymptotically h-expansive ([4]). We choose to skip the definition and instead refer the reader to [2] for all relevant details regarding this notion. We need a result of Boyle et al. ([2]):

An asymptotically h-expansive system has a principal symbolic extension.

Taking the above into account we see that $Z$ has a symbolic principal extension which we call $Y^{\prime}$; here the extension map is $f$. It is obvious that $Y^{\prime}$ is a principal symbolic extension of $Y$. The final situation can now be depicted by the following diagram:

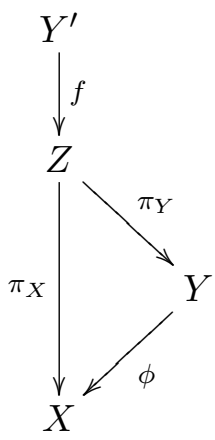

The proof is complete, as the composition $\psi:=\pi_{X} \circ f$ realizes the desired continuous extension of $X$.

\section{References}

[1] M. Boyle and T. Downarowicz, The entropy theory of symbolic extensions, Invent. Math. 156 (2004), 119-161. 
[2] M. Boyle, D. Fiebig and U. Fiebig, Residual entropy, conditional entropy and subshift covers, Forum Math. 14 (2002), 713-757.

[3] M. Denker and M. Keane, Almost topological dynamical systems, Israel J. Math. 34 (1979), 139-160.

[4] F. Ledrappier, A variational principle for the topological conditional entropy, in: Lecture Notes Math. 729, Springer, 1979, 78-88.

[5] M. Misiurewicz, Diffeomorphism without any measure of maximal entropy, Bull. Acad. Polon. Sci. 21 (1973), 903-910.

Jacek Serafin

Institute of Mathematics and Computer Science

Wrocław University of Technology

Wybrzeże Wyspiańskiego 27

50-370 Wrocław, Poland

E-mail: jacek.serafin@pwr.wroc.pl

Received 23 February 2009;

in revised form 28 April 2009 